\title{
Equilibrium cluster formation in concentrated protein solutions and colloids
}

\author{
Anna Stradner 1, Helen Sedgwick 2, Frédéric Cardinaux 1, Wilson C. K. Poon 2, Stefan U. Egelhaaf 2,3 \& Peter \\ Schurtenberger 1 \\ 1 Department of Physics, University of Fribourg, Chemin du Musée3, $\mathrm{CH}-1700$ Fribourg, Switzerland \\ 2 School of Physics and COSMIC, The University of Edinburgh, Mayfield Road, Edinburgh EH9 3JZ, UK \\ 3 School of Chemistry, The University of Edinburgh, West Mains Road, Edinburgh EH9 311, UK
}

Controlling interparticle interactions, aggregation and cluster formation is of central importance in a number of areas, ranging from cluster formation in various disease processes to protein crystallography and the production of photonic crystals. Recent developments in the description of the interaction of colloidal particles with short-range attractive potentials have led to interesting findings including metastable liquid-liquid phase separation and the formation of dynamically arrested states (such as the existence of attractive and repulsive glasses, and transient gels) ${ }^{1-7}$ The emerging glass paradigm has been success-fully applied to complex soft-matter systems, such as colloid- polymer systemss and concentrated protein solutions ${ }^{9}$. However, intriguing problems like the frequent occurrence of cluster phases remain ${ }^{10-13}$. Here we report small-angle scattering and confocal microscopy investigations of two model systems: pro-tein solutions and colloid-polymer mixtures. We demonstrate that in both systems, a combination of shortrange attraction and long-range repulsion results in the formation of small equili-brium clusters. We discuss the relevance of this finding for nucleation processes during protein crystallization, protein or DNA self-assembly and the previously observed formation of cluster and gel phases in colloidal suspension ${ }^{12-17}$.

A number of globular proteins have been shown to exhibit the major characteristics of colloids that interact via a short-range attractive potential. At high ionic strength, where the salt screens electrostatic repulsions, these short-range attractions increasingly dominate with decreasing temperature. This leads to a metastable liquid-liquid phase separation and related critical phenomena ${ }^{18-20}$. In agreement with predictions from modecoupling theory ${ }^{9}$, there is also-evidence for a glass or gel transition at low-particle volume fractions and high interparticle attractions. Such a scenario obviously affects the ability to form the high quality crystals required for protein crystallography ${ }^{15}$.Using two apparently quite different model systems, we demonstrate the generality of this emerging description of the effect of a short-range attraction combined with either a hard or soft repulsion on the phase behaviour of a wide range of colloidal suspensions.

We first investigated solutions of the globular protein lysozyme (molecular mass $14.4 \mathrm{kDa}$, radius $\mathrm{R}_{\mathrm{m}} \approx 1.7$ $\mathrm{nm}){ }^{17-19}$.Using small- angle X-ray (SAXS) and neutron (SANS) scattering, we studied spatial correlations in concentrated solutions at low ionic strength, where the long-range repulsive electrostatic potential is only weakly screened. We then compared these findings with confocal microscopy results using colloid-polymer mixtures, a popular model system with easily tunable interactions. Here we used spherical colloidal particles interacting with a long-range repulsion resulting from a modest charge ${ }^{21}$ and a short-range attraction induced by a polymer-mediated 'depletion effect' ${ }^{22}$.

Figure 1 presents SAXS measurements on a lysozyme concen- tration series at $5{ }^{\circ} \mathrm{C}$. The normalized scattering intensities $\mathrm{I}(\mathrm{q}) / \mathrm{c}$, where $\mathrm{I}(\mathrm{q})$ is the scattering intensity at scattering vector $\mathrm{q}$, and $\mathrm{c}$ is the protein concentration, show a forward intensity $\mathrm{I}(\mathrm{q} \rightarrow 0) / \mathrm{c}$ that 

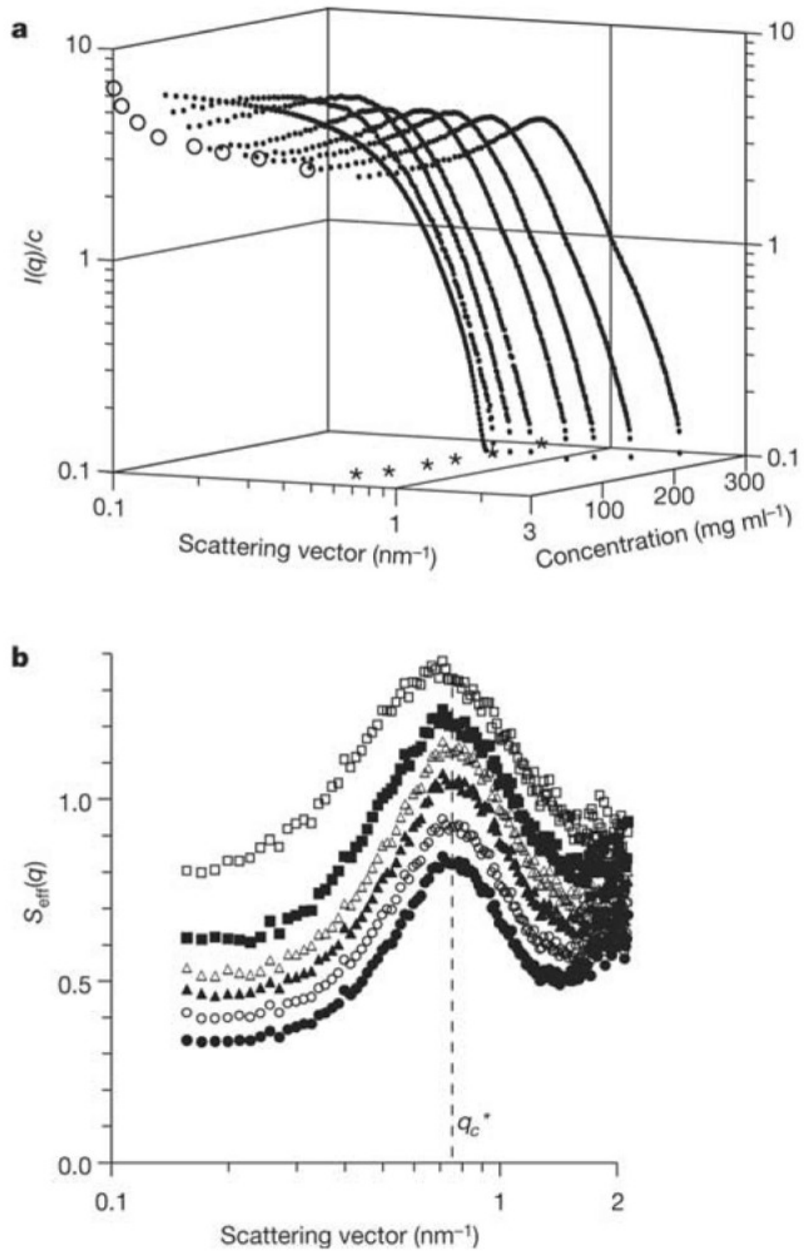

owing to the decreased osmotic compressibility.
Figure 1 Normalized scattered intensity I(q)/c and corresponding effective structure factors $S_{\text {eff }}(q)$,as obtained by SAXS from lysozyme solutions of different concentrations c. a, I(q)/c of a dilution series $(3 \mathrm{mg} / \mathrm{ml}$ to $273 \mathrm{mg} / \mathrm{ml})$ at $5^{\circ} \mathrm{C}$. Large open circles represent the I(q)/values extrapolated to $\mathrm{q}$ $=0.1 \mathrm{~nm}^{-1}$ and stars show the projection of the peak maximum onto the q-c plane. $\mathbf{b}, S_{\text {eff }}(q)$ of the concentrated samples in a. Concentration ranges from $36 \mathrm{mg} / \mathrm{ml}$ (open squares) to $273 \mathrm{mg} / \mathrm{ml}$ (filled circles).

decreases with increasing concentration (Fig. la). This indicates strongly repulsive interactions, in contrast to efficiently screened lysozyme solutions for which I(q $\rightarrow \mathrm{O}) / \mathrm{c}$ increases because the short-range attractions are dominant9. At the same time a pro-nounced peak at finite q evolves, indicating strong positional correlations between the proteins.

Further information about these correlations comes from the effective structure factor $S_{\text {eff }}(q)$, which is obtained by dividing $I(q) / c$ by the normalized intensity of a very dilute sample. Qualitatively, a peak in $S_{\text {eff }}(q)$ at $q^{*}$ can be interpreted as a 'Bragg reflection' from planes of particles separated by the mean nearest-neighbour distance $\mathrm{d}$, with $\mathrm{d} \approx 2 \pi / \mathrm{q}^{*}$. Charged colloids at low ionic strength maximize their average interparticle distance, and so $\mathrm{d}$ depends on the volume fraction $\Phi$ and we find $\mathrm{q}^{*} \approx 2 \pi \mathrm{n}^{1 / 3}$, where $\mathrm{n}=3 \Phi /\left(4 \pi \mathrm{R}^{3}\right)$ is the number density of particles with radius $\mathrm{R}$. At the same time the forward scattering becomes suppressed upon increasing $\Phi$,

Surprisingly, the peak position $\mathrm{q}_{\mathrm{c}}{ }^{*}$ of $\mathrm{S}_{\mathrm{eff}}(\mathrm{q})$ is essentially independent of $\Phi$ (Fig. lb). Moreover, there appears to be an upturn at high q values. SANS measurements over an extended range of q values indeed show the existence of a second peak in $\mathrm{S}_{\mathrm{eff}}(\mathrm{q})$ (Fig. 2). The position $\mathrm{q}_{\mathrm{m}}{ }^{*}$ and height of this second peak changes neither with concentration nor with temperature. The position $\mathrm{q}_{\mathrm{c}}{ }^{*}$ of the low-q peak is also independent of concentration, in agreement with our SAXS results (Fig. lb), but it does depend on temperature and shifts to lower q values with decreasing temperature.

These unexpected observations can be understood if the proteins self-assemble into small clusters with a $\Phi$ dependent aggregation number $\mathrm{N}_{\mathrm{c}}$. The driving force for self-assembly into clusters is short-range attraction, which effectively acts as surface tension leading to a decrease in surface energy upon aggregation. On the other hand, cluster growth is limited by the increasing electrostatic energy of the clusters, which counterbalances the gain in surface energy and is due to the small number of residual charges combined with a low ionic strength. A low ionic strength ensures that the Debye length is larger or comparable to the cluster size. 


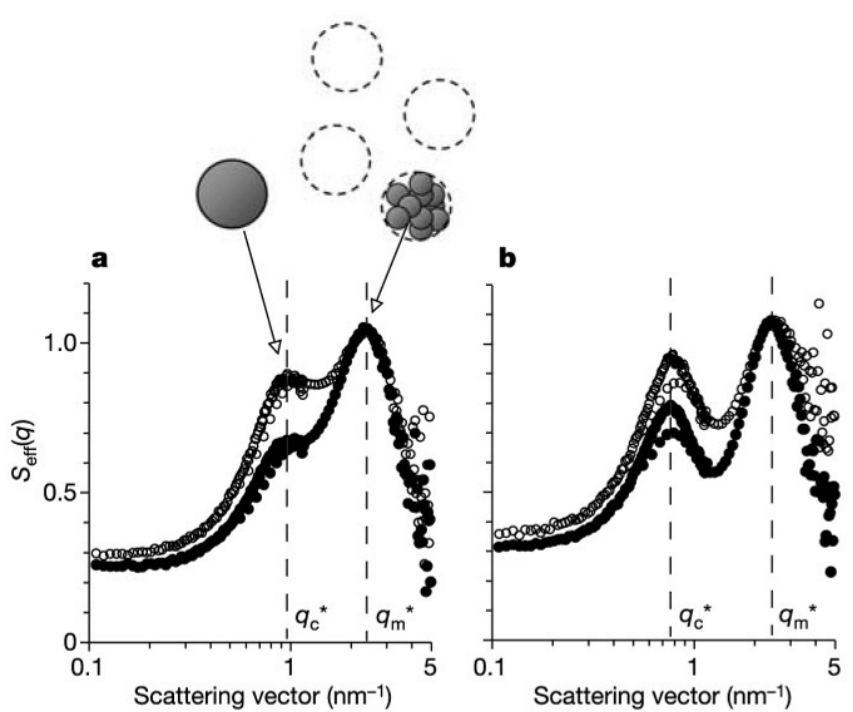

Figure 2 Effect of concentration and temperatureon the effective structure factor $S_{\text {eff }}(q)$ as obtained by SANS.

a, $254 \mathrm{mg} / \mathrm{ml}$ (filled symbols) and $169 \mathrm{mg} / \mathrm{ml}$ (open symbols) lysozyme solutions at $25^{\circ} \mathrm{C}$. b, The same samples at $5^{\circ} \mathrm{C}$. The dashed lines highlight that both peak positions are independent of lysozyme concentration. The second peak (corresponding to internal monomermonomer correlations within the dense particle clusters) changes neither with concentration nor with temperature. The cluster-cluster correlation peak at lower $\mathrm{q}$ is also concentration independent but shows a strong temperature dependence, indicating fewer but larger aggregates at lower temperatures.

This balance etween short-range attraction and weakly screened (and thus long-range) electrostatic repulsion provides a stabilizing mechanism against gelation and determines a finite aggregation number $\mathrm{N}_{c}$, as in micelle formation. Equilibrium cluster formation in colloidal suspensions has recently been investigated theoretically and supported by computer simulations ${ }^{10-11}$. Moreover, a theoretical model has previously been proposed for mesophase separation of colloids in organic solvents and an explicit expression for the concentration dependence of $\mathrm{N}_{\mathrm{c}}$ has been derived ${ }^{12}$.

This model allows us to assign the first peak in $\mathrm{S}_{\text {eff }}(\mathrm{q})$ at $\mathrm{q}_{\mathrm{c}}{ }^{*}$ (Fig. 2a) to cluster-cluster correlations caused by electrostatic interactions between the charged clusters, whereas the second peak at $\mathrm{q}_{\mathrm{m}}{ }^{*}$ reflects the positional correlations of the monomers within a single cluster. This interpretation is directly supported by numerical simulations ${ }^{10-11}$. The monomer peak at $\mathrm{q}_{\mathrm{m}}{ }^{*}$ exhibits a concentration and temperature independent value $\mathrm{q}_{\mathrm{m}}{ }^{*} \mathrm{R}_{\mathrm{m}} \approx 3.8$, indicating a constant packing density within the cluster of approximately $60 \%$ by volume ${ }^{23}$. Also the position $\mathrm{q}_{\mathrm{c}}{ }^{*}$ of the cluster-cluster peak is independent of $\Phi$ at higher concentrations, which implies a constant cluster number density $\mathrm{n}_{\mathrm{c}}$ (because $\mathrm{q}_{\mathrm{c}}{ }^{*} \propto \mathrm{n}_{\mathrm{c}}{ }^{1 / 3}$ ). This allows us to determine the dependence of $\mathrm{N}_{\mathrm{c}}$ on $\Phi$ according to $\mathrm{Nc}=\mathrm{n}_{\mathrm{m}} / \mathrm{n}_{\mathrm{c}} \Phi / \mathrm{n}_{\mathrm{c}}$, where $\mathrm{n}_{\mathrm{m}}$, is the monomer number density, and the proportionality constant has been determined from a comparison with calculations using the Rogers-Young closure relation ${ }^{24}$. We find $\mathrm{Nc} \infty \Phi$, in agreement with the theoretical prediction ${ }^{12}$. This is not obvious for such small clusters and high volume fractions where we would expect to find considerable contributions to the free energy from both (translational) entropy and from cluster-cluster interactions. However, the entropy con-tribution that would favour the formation of even smaller clusters may largely be compensated for by the curvature dependence of the interfacial tension. Small clusters have a relatively large number of surface proteins with unfavourable 'broken contacts', and surface tension may thus increase correspondingly.

From $\mathrm{q}_{\mathrm{c}}{ }^{*}$ and $\Phi$, absolute values of an average $\mathrm{N}_{\mathrm{c}}$ can be estimated, using the above relationships (Fig. 3a). The effect of temperature can be understood from the subtle balance of opposing forces; decreasing temperature increases the attraction ${ }^{18}$ and hence leads to larger clusters and smaller $\mathrm{n}_{\mathfrak{c}}$, which in turn decreases $\mathrm{q}_{\mathrm{c}}{ }^{*}$. It is important to point out that the temperature-dependent cluster formation is fully reversible, which strongly supports the idea of equilibrium cluster formation.

Decreasing the temperature shifts the balance in favour of attractive interactions, but a similar effect is also expected following the reduction of electrostatic repulsion by increasing the salt concentration. We did indeed observe this (Fig. 4). The first peak of $\mathrm{S}_{\text {eff }}(\mathrm{q})$ (filled circles) is shifted to a lower q value when the temperature is decreased (open circles) or the salt concentration is increased (open squares). We estimate $\mathrm{N}_{\mathrm{c}} \approx 3$ at $20^{\circ} \mathrm{C}$ 
without salt, and $\mathrm{Nc} \approx 5$ when either the temperature is decreased to $5^{\circ} \mathrm{C}$ or $50 \mathrm{mM} \mathrm{NaCl}$ is added. However, temperature and salt are not completely equivalent; salt also screens cluster-cluster interactions, which leads to enhanced forward scattering (Fig. 4). This demon-strates that the subtle balance between short-range attraction and weakly screened Coulomb repulsion allows us to tune the aggregation behaviour and the resulting cluster sizes.

With a further increase in salt concentration, the screening length will eventually become significantly smaller than the cluster size. The electrostatic energy can then no longer stabilize the clusters, leading to irreversible aggregation and precipitation. At $50 \mathrm{mM} \mathrm{NaCl}$, the Debye length is approximately $1.3 \mathrm{~nm}$, and we indeed observe amorphous precipitates at temperatures below $15^{\circ} \mathrm{C}$ (Fig. 4, inset).
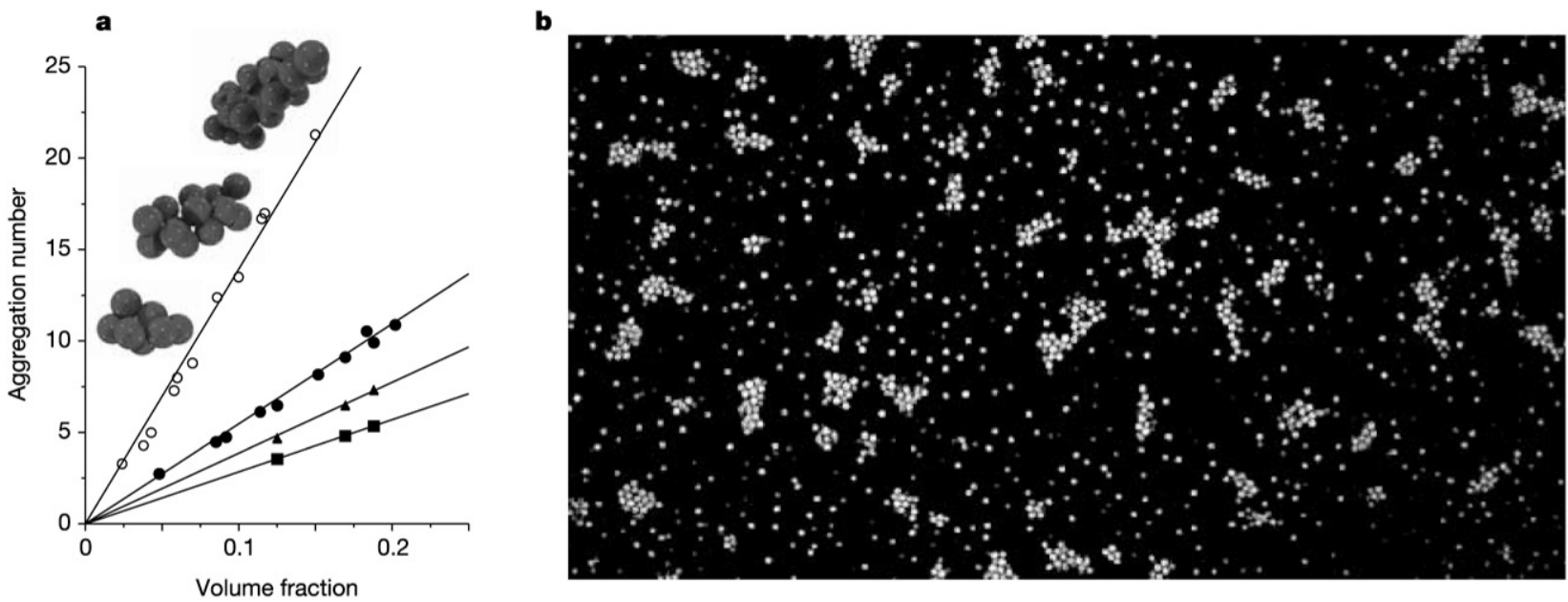

Figure 3 Clusters in protein solutions and colloidal suspensions. a, Average aggregation number of clusters $\left(\mathrm{N}_{\mathrm{c}}\right)$ versus volume fraction $(\Phi)$ in lysozyme samples at $5{ }^{\circ} \mathrm{C}$ (filled circles), $15^{\circ} \mathrm{C}$ (triangles) and $25^{\circ} \mathrm{C}$ (squares), and in colloidpolymer mixtures ( $c_{p} \approx 3 \mathrm{mg} / \mathrm{ml}$, open circles). Values for lysozyme samples were determined using SAXS, and confocal microscopy was used for colloid-polymer mixtures. The insets show typical clusters plotted using real particle coordinates from confocal microsopy experiments on colloid-polymer mixtures $\left(c_{p} \approx 3 \mathrm{mg} / \mathrm{ml} \Phi=0.024,0.086\right.$ and 0.15$)$. b, confocal microscopy image from a colloid-polymer mixture $\Phi=0.086, c_{p} \approx 3 \mathrm{mg} / \mathrm{ml}$ ).

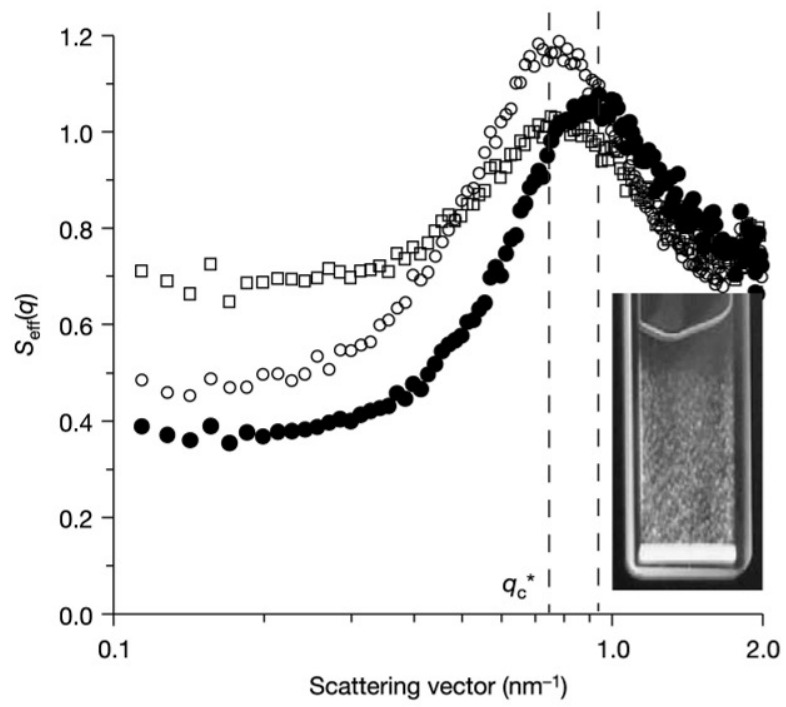

Figure 4 Effect of temperature and ionic strength on the effective structure factor $S_{\text {eff }}(q)$, obtained by SAXS. $125 \mathrm{mg} / \mathrm{ml}$ lysozyme solution without salt (open and filled circles) and with $50 \mathrm{mM} \mathrm{NaCl}$ (open squares). The filled circles and open squares represent data obtained at 20 " $\mathrm{C}$, the open circles show data obtained at $5{ }^{\circ} \mathrm{C}$, The dashed lines highlight that increasing the ionic strength or decreasing the temperature both shift the cluster-cluster peak to lower q values. The inset shows the onset of precipitation in a sample with $50 \mathrm{mM} \mathrm{NaCl}$ when the temperature is decreased below $15^{\circ} \mathrm{C}$. 
There are numerous reports of finite size clusters or 'aggregates' of unknown origin in biological and colloidal systems; these include aggregate formation in insulin 16, the formation of almost mono-disperse mixed aggregates of polyelectrolytes (such as DNA) and surfactants ${ }^{14}$, and the existence of pre-nucleation clusters in crystallizing protein solutions ${ }^{17}$. We speculate that it is the delicate balance between short-range attractive and electrostatic repulsive forces that leads to this controlled self-assembly.

To explore the general validity of this concept across a broad range of systems and to observe these equilibrium clusters directly, we investigated a colloidal model system using confocal microscopy. We used density-matched, hard-sphere colloidal particles (radius $660 \mathrm{~nm}$ ) with a modest charge and a short-range (about $2 \%$ of the particle diameter) attraction induced by a polymer-mediated 'depletion effect'.

As the polymer concentration $\mathrm{c}_{\mathrm{p}}$ (and therefore the strength of the attraction) is increased, a transition from an ergodic fluid (in which individual particles undergo brownian motion) to clustering occurs. Thereafter, over an intermediate range of $c_{p}$, values, indi-vidual mobile clusters exist (Fig. $3 b$ ). At low $\Phi$, the clusters coexist with single particles, whereas at high $\Phi$ monomers are less frequent. These states of cluster-monomer coexistence seem to be long-lived: clusters were not observed to grow by further aggregation. At even higher $\mathrm{c}_{\mathrm{p}}$, gelation occurs.

Figure 3a shows the average aggregation number of clusters, $\mathrm{N}_{\mathrm{c}}$, as a function of colloid volume fraction $\Phi$ at $\mathrm{C}_{\mathrm{p}} \approx 3 \mathrm{mg} / \mathrm{ml}$, which corresponds to a contact attraction between neighbours of about $5 K_{B} \mathrm{~T}$, where $K_{B}$ is Boltzmann's constant. As for the protein samples, an $\mathrm{Nc} \propto \Phi$ scaling is found, which agrees with the theoretical model and computer simulations ${ }^{11-12}$. Moreover, the absolute values are consistent with the system parameters $^{12}$. $\mathrm{N}_{\mathrm{c}}$ was furthermore observed to rise slightly when $\mathrm{c}_{\mathrm{p}}$ was increased, which is equivalent to deepening the interparticle attraction; this is in agreement with the temperature dependence found for the protein solutions.

Our results from two very different systems unambiguously confirm that the formation of equilibrium clusters in protein solutions is not caused by protein-specific interactions; rather, the combination of a weakly screened, long-range electrostatic repulsion and a short-range attraction leads to the formation of small equilibrium clusters with a concentration-dependent aggregation number $\mathrm{N}_{\mathrm{c}}$. Our findings demonstrate the general importance of residual, weakly screened charges together with short-range attractions in cluster formation, and suggest a possible means of obtaining tunable cluster formation through protein or colloid selfassembly. This mechanism might also provide an alternative route to nanostructured colloidal gel and glass phases, where the structural elements could be self-assembled colloid clusters ${ }^{11}$.

\section{Methods}

\section{Preparation of protein solutions}

Hen egg white lysozyme was obtained from Fluka (L7651,three times crystallized, dialysed and lyophilized) and used without further purification. About 40mg protein was dissolved per millilitre of a 20 mM HEPES buffer in $\mathrm{D}_{2} \mathrm{O}(99.9 \%$, Cambridge Isotope Laboratories) at $\mathrm{pH} 7.8$, where the lysozyme carries a net positive charge of about eight electronic charge ${ }^{19,25}$. This stock solution was stirred at room temperature and passed through a $0.22-\mu \mathrm{m}$ filter to remove any undissolved material. An Amicon ultrafiltration stirring cell with a YM-10 membrane was used to wash the protein solution with buffer (to reduce any ionic impurities left) and to further concentrate it. At the highest lysozyme concentrations, the $\mathrm{pH}$ had slightly increased to values between 8.0 and 8.2, corresponding to a charge uncertainty of $\pm 2 \%\left({ }^{25}\right)$. Control experiments using samples prepared under strict $\mathrm{pH}$ control $(\mathrm{pH}$ values for all concentrations were $7.8 \pm 0.1)$ gave the same results as the 
samples with small $\mathrm{pH}$ variations, thus indicating that the small variations in the effective net charge have no measurable influence on the cluster formation mechanism (see Supplementary Information). We also checked the temperature dependence of the $\mathrm{pH}$ for a concentrated lysozyme solution and found an increase of only 0.2 units when decreasing the temperature from 33 to $5^{\circ} \mathrm{C}$. Again, this results in a negligible charge variation when looking at the titration curve ${ }^{25}$. Lower concentrations were prepared by diluting the stock solution with buffer at $\mathrm{pH}$ 7.8. The samples with $50 \mathrm{mM} \mathrm{NaCl}$ were obtained by diluting a concentrated protein sample with HEPES buffer containing the appropriate amount of $\mathrm{NaCl}$ at $\mathrm{pH} 7.8$. The final concentrations were determined by ultraviolet absorption spectroscopy at $280 \mathrm{~nm}$ using a specific absorption coefficient $\mathrm{E}_{1 \mathrm{~cm}}{ }^{1 \%}=26.4$; the highest concentrations were typically between 250 and $350 \mathrm{mg} / \mathrm{ml}$. Using a partial specific volume of $0.74 \mathrm{~cm} 3 / \mathrm{g}$ for the proteins results in the corresponding protein monomer volume fractions of $0.185<=\Phi<=0.26$.

\section{Preparation of colloid-polymer mixtures}

Spherical particles (radius $\mathrm{R}=660 \mathrm{~nm}$ ) with polymethylmethacrylate (PMMA) cores fluorescently labelled with nitrobenzoxadiazole and sterically stabilized by a thin $(\approx 10 \mathrm{~nm})$ layer of chemically grafted poly-12hydroxystearic acid were suspended in an approximately 1:4 mixture of cis-decalin and cycloheptyl bromide (CHB) for density matching. CHB leads to a positive charge $\mathrm{Q}$ of the PMMA particles ${ }^{21}$ with $\mathrm{Q}<=10^{3}$ electronic charges for the present particles (estimated from $\Phi$ at crystallization). Addition of linear, nonadsorbing polystyrene (Polymer Laboratory, molecular mass $212.4 \mathrm{kDa}$ ) induces an effective attraction between the PMMA particles: exclusion of polymer between the surfaces of two nearby particles results in a net osmotic force that pushes them together ${ }^{22}$. The depth and range of this 'depletion' attraction are proportional to the polymer concentration and polymer size, respectively. Polymer size can be estimated by twice the radius of gyration $r_{g}$, of a single coil, giving a dimensionless range $\delta \approx r_{\mathrm{g}} / R$, here $\delta \approx 0.02$.

\section{Small-angle X-ray scattering measurements}

SAXS experiments were carried out with a pinhole camera (NanoSTAR, Bruker AXS) equipped with a sealed tube $(\mathrm{Cu} \mathrm{K} \alpha)$, a thermostatically regulated sample chamber and a two-dimensional gas detector. The $\mathrm{q}$ range is $0.1-2 \mathrm{~nm}^{-1}$.

\section{Small-angle neutron scattering measurements}

SANS experiments were performed at the SANS I facility at the Swiss neutron source SINQ at the Paul Scherrer Institut, Switzerland. We used $1-\mathrm{mm}$ and 2-mm Hellma quartz cells and a thermostatically regulated sample holder. Combinations of different wavelengths (5 and $8 \AA$ ), sample-to-detector distances (1.6-18 m) and collimation lengths $(4.5-18 \mathrm{~m})$ were used to cover a $\mathrm{q}$ range of $0.1-7 \mathrm{~nm}^{-1}$.

\section{Confocal microscopy}

Imaging was carried out in the Collaborative Optical Spectroscopy, Micromanipulation and Imaging Centre (COSMIC). A small amount of sample was sandwiched between a cover slip and a microscope slide. The sample thickness was fixed at about $0.3 \mathrm{~mm}$ by spacers. A Nikon TE-300 with a Biorad Radiance 2100MP scanning head was used; fluorescence was excited at $488 \mathrm{~nm}$ and observed at $525 \mathrm{~nm}$.

doi:10.1038/nature03109. 
1. Dawson, K. A. The glass paradigm for colloidal glasses, gels, and other arrested states driven by attractive interactions. Curr.Opin. Colloid Interf. Sci. 7, 218-227 (2002).

2. Trappe, V., Prasad, V., Cipelletti, L., Segre, P. N. \& Weitz, D. A. Jamming phase diagram for attractive particles. Nature 411, 772-775 (2001).

3. Sciortino, F. Disordered materials: one liquid, two glasses. Nature Mater. 1, 145-146 (2002).

4. Pham, K. N. et al. Multiple glassy states in a simple model system. Science 296, 104-106 (2002).

5. Eckert, T. \& Bartsch, E. Re-entrant glass transition in a colloid-polymer mixture with depletion attractions. Phys. Rev. Lett. 89, 125701-125704 (2002).

6. Weeks, E. R., Crocker, J. C., Levitt, A. C., Schofield, A. \& Weitz, D. A. Three-dimensional direct imaging of structural relaxation near the colloidal glass transition. Science 287, 627-631 (2000).

7. Foffi, G. et al. Phase equilibria and glass transition in colloidal systems with short-ranged attractive interactions: application to protein crystallization. Phys Rev. E 65,031407431417 (2002).

8. Bergenholtz, J., Poon, W. C. K. \& Fuchs,M. Gelation in model colloid-polymer mixtures. Langmuir 19, 4493-4503 (2003).

9. Kulkarni,A. M., Dixit, N. M. \& Zukoski,C. F. Ergodic and non-ergodic phase transitions in globular protein suspensions. Faraday Discuss. 123, 37-50 (2003).

10. Puertas, A. M., Fuchs, M. \& Cates, M. E. Dynamical heterogeneities close to a colloidal gel. J. Chem. Phys. 121, 2813-2822 (2004).

11. Sciortino, F., Mossa, S., Zaccarelli, E. \& Tartaglia, P. Equilibrium cluster phases and low-density arrested disordered states: The role of short-rangeattraction and long-range repulsion. Phys. Rev. Lett. 93,055701 (2004).

12. Groenewold, J. \& Kegel, W. K. Anomalously large equilibrium clusters of colloids. J. Phys. Chem. B 105, 11702-11709 (2001).

13. Segrgé, P. N., Prasad, V., Schofield,A. B. \& Weitz, D. A. Glasslike kinetic arrest at colloidal-gelation transition. Phys. Rev. Lett. 86,6042-6045 (2001).

14. Guillot, S., Delsanti,M., Desert, S. \& Langevin,D. Surfactant-induced collapse of polymer chains and monodisperse growth of aggregates near the precipitation boundary in carboqmethylcellulose- DTAB aqueous solutions. Langmuir 19,230-237 (2003).

15. Muschol, M. \& Rosenherger, F. Liquid-liquid phase separation in supersaturated lysozyme solutions and associated precipitate formation/crystallization. J. Chem. Phys. 107, 1953-1962 (1997).

16. Pedersen,J. S., Hansen, S. \& Bauer, R. The aggregation behavior of zinc-free insulin studied by smallangle neutron scattering. Eur. Biophys.J. 22,379-389 (1994).

17. Piazza, R. Interactions and phase transitions in protein solutions. Curr.Opin. Colloid Interf. Sci. 5, 3843 (2000).

18. Malfois, M., Bonnete, F., Belloni, L. \& Tardieu, A. A model of attractive interactions to account for fluid-fluid phase separation of protein solutions. J. Chem. Phys. 105,3290-3300 (1996).

19. Broide, M. L., Tomic, 1. M. \& Saxowsky, M. D. Using phase transitions to investigate the effect of salts on protein interactions. Phys. Rev. E53,6325-6335 (1996).

20. Schurtenherger, P., Chamherlin, R. A., Thurston, G. M., Thomson, J. A. \& Benedek,G. B. Observation of critical phenomena in a pratein-water solution. Phys. Rev. Len 63, 2064-2067 (1989).

21. Yethiraj, A. \&Van Blaaderen, A. A colloidal model system with an interaction tunable from hard sphere to soft and dipolar. Nature 421, 513-517 (2003).

22. Poon, W. C. K. The physics of a model colloid-polymer mixture. J. Phys. Condens. Matter 14, R859R880 (2002).

23. Pham, K. N., Egelhaaf, S. U., Pusey, P. N. \& Poon, W. C. K. Glasses in hard spheres with short-range attraction. Phys. Rev. E 69, 11503-1 1516 (2004).

24. Rojas, L., Urban, C., Schurtenherger, P., Gisler, T.\& Grünherg, H. H. Reappearance of structure in charge-stabilized suspensions. Europhys. Lett. 60,802-808 (2002). 
25. Tanford, C. \& Roxby, R. Interpretation of protein titration curves. Application to lysozyme. Biochemistry 11, 2192-2198 (1972).

\section{Acknowledgements}

We thank the Swiss spallation source at the Paul Scherrer Institut (PSI) in Villigen, Switzerland, for the neutron beam time and we acknowledge the help of our local contacts J. Kohlbrecher and S. van Petegem. We thank J. Groenewold, W. Kegel, F. Sciortino, K. Kroy and M. Cates for discussions. We thank A. Schofield for preparing the fluorescent PMMA particles. This work was supported by the Swiss National Science Foundation, the UK Engineering and Physical Sciences Research Council, the Scottish Higher Education Funding Council, and the Marie Curie Network on Dynamical Arrest of Soft Matter and Colloids. A.S. and P.S. conceived and performed the protein experiments; F.C. prepared the $\mathrm{pH}$ stabilized protein samples for the control experiments; H.S., W.C.K.P. and S.U.E. carried out and analysed the experiments with the colloid-polymer samples.

Correspondence and requests for materials should be addressed to P.S. (peter.schurtenberger@unifr.ch). 